\title{
OBSERVATIONS OF GALACTIC AND EXTRAGALACTIC JETS
}

\author{
R. A. PERLEY
}

National Radio Astronomy Observatory

Socorro, N.M., 87801

\section{I) INTRODUCTION}

Since nearly all discrete radio sources of astronomical interest are of insufficient angular extent for their detailed structural properties to be accessible to singledish radio telescopes, radio interferometry must be employed to gain information on the morphologies of these objects. Recently constructed imaging interferometer arrays which employ the technique of Fourier synthesis, particularly MERLIN and the VLA (Very Large Array), and the more recent VLBI arrays, have given unprecedented imaging capabilities, with the result that our knowledge, and hence perceptions, of discrete radio sources have vastly changed over the last few years. An equally important parallel development has been image processing algorithms. These have vastly improved the quality of information produced by these arrays, so that an instrument such as the VLA can now produce images with speed and quality exceeding original design specifications by factors of 100 to 1000 .

In this contribution, the current state of observations of the phenomenon of radio jets, both galactic and extragalactic is reviewed. Although the bulk of the observations are from radio wavelengths, optical and X-ray data will be used where available. Due to space considerations, only a cursory review is possible and, since the following two talks will deal with interpretation, the review will be concerned almost solely with the data. Readers wishing more detail should consult the recent review articles by MILEY [1], on the structure of extended extragalactic radio sources; by BRIDLE and PERLEY [2], on extragalactic jets; and by KELLERMANN and PAULINY- TOTH [3], on compact radio sources. The theory of extragalactic jets is reviewed by BEGELMAN, BLANDFORD and REES [4]. The proceedings of two recent workshops $[5,6]$ are also recommended.

The review begins with stellar jets, but as the majority of the available data concerns extragalactic jets, the bulk of this paper will be concerned with this phenomenon. For these, I will start with a description of the overall morphology of extragalactic radio sources, whose scales can exceed $1 \mathrm{Mpc}$, and work towards the pc scales accessible with VLB techniques. Although this range of 1 million is impressive, it is still far less than the 12 orders of magnitude on which the extragalactic jet phenomenon is expected to operate over, as described by Roger Blandford in his review talk. Thus the physics of the central engine lie far inside those scales currently accesible to direct observation.

\section{II) STELLAR JETS}

Jets, or features which are suggestive of jets, are being found with increasing frequency amongst members of two very different classes of stellar objects. The first, protostellar objects, has been reviewed by Reinhart Mundt in the first talk of this colloquium. As noted by him, these jets are associated with pre-main sequence objects, notably T-Tauri stars, and are found through Ha searches of star-forming regions. These jets are supersonic, and have lengths of typically .01 to .2 pc, opening angles of 3 to 10 degrees, and velocities of 50 to $400 \mathrm{Km} / \mathrm{sec}$. Their termination or deflection points are suspected of being Herbig-Haro (HH) objects, which are thus explained in terms of shock heating from deceleration of the flow. Many of these jets are found in the same regions as bipolar molecular outflows [7], and it seems probable that a close relation exists between these phenomena. A recent review by LADA [8] is recommended.

The second class of stellar jets is associated with late-type objects. Most suspected or known jets in this class are X-ray sources, and their observational parameters have been recently reviewed [9], and are summarised: 
a) SS433. This most interesting object, a binary system with 13-day period, has been the object of intense observational efforts since the discovery of the extraordinary, time-variable Doppler shifted Balmer and He I emission lines. The observations have been reviewed by MARGON [10]. The widely accepted kinematic model employs oppositely-directed twin gas jets of velocity $0.26 \mathrm{c}$ which precess with a period of approximately 164 days with half-angle of 20 degrees about a central axis inclined by 79 degrees to the line of sight. Both the optical and radio data are fitted excellently by this model. The arcsecond scale radio maps $[9,11]$ show projected corkscrews with knots whose motion appears to be purely ballistic. There is no apparent evolution of the spectral index, $\alpha$, (defined as $\log I=$ const $+\alpha \log \nu$ ) of -0.6 to -0.7 , and no depolarization down to $20 \mathrm{~cm}$ wavelength. No attempt to measure the rotation measure (RM) has yet been made, hence the intrinsic magnetic field directions are unknown. The blobs' apparent motion of 3 " per year allow independent estimates of the velocity, and give a firm distance estimate of $5.5 \mathrm{Kpc}$ [12].

b) Cyg X-3. Flux density measurements of this object, which is a binary with 4.8 hour period, but whose identification is uncertain, show violent flares [13]. Recent radio observations $[14,15]$ show evidence of rapid $(\sim 0.1 \mathrm{c})$, one-dimensional expansion on sub-arcsecond scales. The small angular size ( $\leq 0.1$ arcsec) prevents detailed tracking of the structure, so the evidence that this object is a jet is weak, but promising.

c) Sco $\mathrm{X}-1$. This object is an extragalactic look-alike, with a core and pair of compact knots [16]. Recent VLA observations [17] show that the entire source is moving southward at $70 \mathrm{~km} / \mathrm{sec}$. The northern hotspot is moving radially outward at 35 $\mathrm{km} / \mathrm{sec}$, relative to the core. The southern hot spot is weaker, and an upper limit to its expansion velocity is $70 \mathrm{~km} / \mathrm{sec}$. Arguing by analogy with extragalactic sources, it seems likely that a relativistic jet is present [16], although there is no direct observational evidence of one.

d) $\mathrm{CH}$ Cyg. This object is a symbiotic star, and the evidence for a jet is fairly good, being presented in a contributed paper to this Colloquium by Taylor and Seaquist. They derive outflow velocities of $\sim 1000 \mathrm{~km} / \mathrm{sec}$. Another symbiotic star, $R$ Aquarii, has elongated structure seen in the radio, optical, and near-uv, which may represent a jet.

\section{III) EXTRAGALACTIC JETS}

Amongst the first objects observed with the newly constructed VLA were those radio galaxies and quasars which showed evidence of elongated structures from lower resolution maps. On theoretical grounds, the most luminous radio galaxies and quasars are expected to be resupplied on a quasi-continuous time scale $[18,19]$, one means of which could be by a highly collimated supersonic flow, or jet [20]. The increased sensitivity and resolution that modern instruments provided over earlier ones soon revealed jets in a large proportion of extragalactic sources; the number of known jets (defined by the criteria in [2]) is now 143 [21], with at least 80 more that probably will join the list when better data are taken.

\section{1) EXTENDED SOURCES}

I discuss here the morphological characteristics of extended extragalactic radio sources. Since virtually all extragalactic sources show extended structure, the definition of an extended source is here made to specify lobe-dominated sources those whose flux density is dominated by optically thin emission with spectral index $(\alpha)$ of -.6 to -.9 .

a) Large-Scale Structure At low resolution, virtually all extended extragalactic sources show collimated, bilateral symmetry about a line which passes near the optical identification, if known. Both structural extent and brightness show symmetry, these can be used to place an upper limit to the bulk velocity of advance of $\sim 0.1 \mathrm{c}$ of the extended regions $[22,23]$.

There is a striking correlation between morphology and radio luminosity [24]. Those sources with $1400 \mathrm{MHz}$ spectral powers exceeding $\sim 10^{25} \mathrm{~W} / \mathrm{Hz}$ are edge-brightened, with regions of intense emission (known as hot spots) at the extremities, and generally linear morphology, while the weaker sources are more diffuse, more distorted, and without hot spots. These differences are illustraed in the following three figures, representing distinct morphological categories. Fig. 1 shows the 
canonical example of a high luminosity source, Cygnus A, showing the hot spots, the diffuse lobes, and the unresolved central core, coincident with a $C D$ galaxy with redshift .0562 [25]. Fig. 2 shows an example of a low luminosity source, 3C449 [26]. The basic double structure is present, but there are no hot spots, and the extended structure extends far beyond the limits of the map. The jets are prominent, emanating from the central unresolved core, which is coincident with the identified galaxy, an elliptical with redshift .0181. Fig. 3 shows an example of a head-tail radio galaxy, NGC1265 [27], a member of the Perseus cluster of galaxies. Head-tail sources are all low-luminosity objects in clusters whose highly bent structures are due to motion of the galaxy through the intra-cluster medium $[28,29]$.

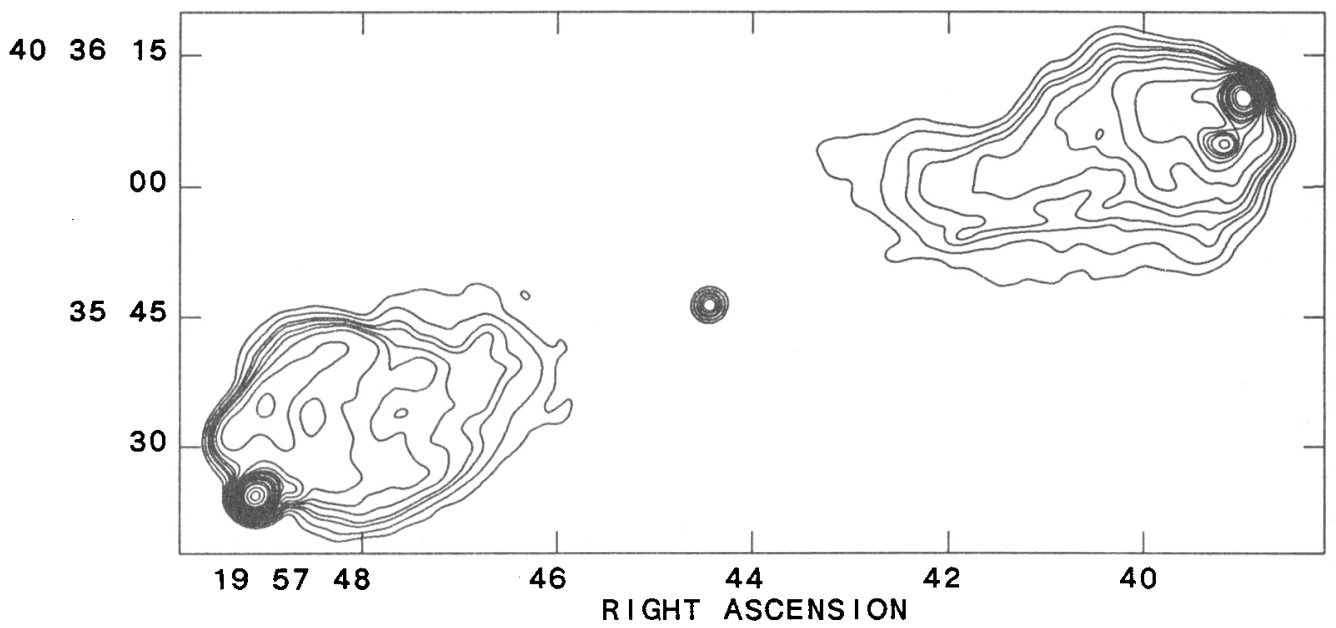

Fig. 1. An image of the prominent high-luminosity radio galaxy Cygnus A at 2 " resolution at $\lambda 6 \mathrm{~cm}$. The contours are drawn at $.2, .4, .75,1,1.5,2,3,5,7.5,10$, $12.5,15,20,25,30,40,50,70$ and $90 \%$ of the maximum.

The emission process in all these extended regions is widely believed to be synchrotron. Assuming this to be so, and using a wel1-known argument [30], the minimum total energies and corresponding magnetic fields can be estimated from observations of the source brightness and size [31]. For high luminosity objects such as Cygnus A (whose radio luminosity between $10 \mathrm{MHz}$ and $10 \mathrm{GHz}$ is $\sim 10^{45} \mathrm{erg} / \mathrm{sec}$ ), the total energy must exceed $10^{59}$ ergs, and the fields in the lobes are typically 60 $\mu$ gauss. For the very large, low luminosity objects, whose typical radio luminosities are $10^{43} \mathrm{erg} / \mathrm{sec}$, the total energies may be in excess of $10^{61}$ ergs, while the corresponding fields may be as low as 1 pgauss. Typical minimum energy densities in relativistic particles and fields in these dilute regions range from $10^{-10}$ to $10^{-13}$ erg $/ \mathrm{cm}^{3}$.

Models of these objects have the lobes being generated from highly collimated jets which form in the nucleus. If the jet is especially energetic, the flow penetrates through the lobe material to the end, terminating in a strong shock in which particle acceleration takes place. In weaker sources, the jet decelerates significantly throughout its travel, perhaps due to entraining material, eventually becoming subsonic. At this point, it is better described as a plume, rather than a momentumdriven jet, and forms the lobes of the source. For high luminosity sources, the lobe advances into the medium, and particles are left behind. In this case, radiation losses can be expected to 'soften' the particle spectra in the older regions of the source - towards the center. For the weak sources, the reverse situation occurs, with the outer regions being the older. Since the particle spectrum is reflected in the radiation spectrum, direct measures of these aging effects can be made, and they generally (though not in detail) support the scenarios drawn above. In Fig. 4 is shown the $6-20 \mathrm{~cm}$ spectral index of Cygnus A. Note the spectral steepening in the inner regions of the source. It must be noted, however, that there are luminous 
sources in which no spectral index gradient is seen [25]; this may be evidence for continuing particle acceleration throughout the lobes.

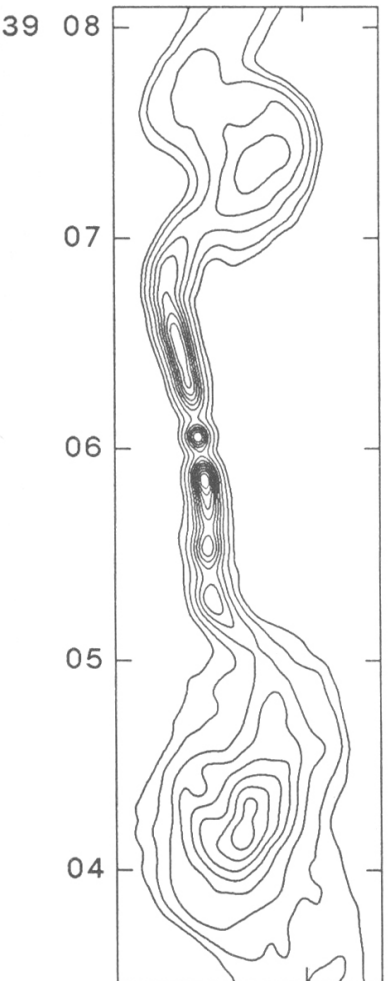

222905
41

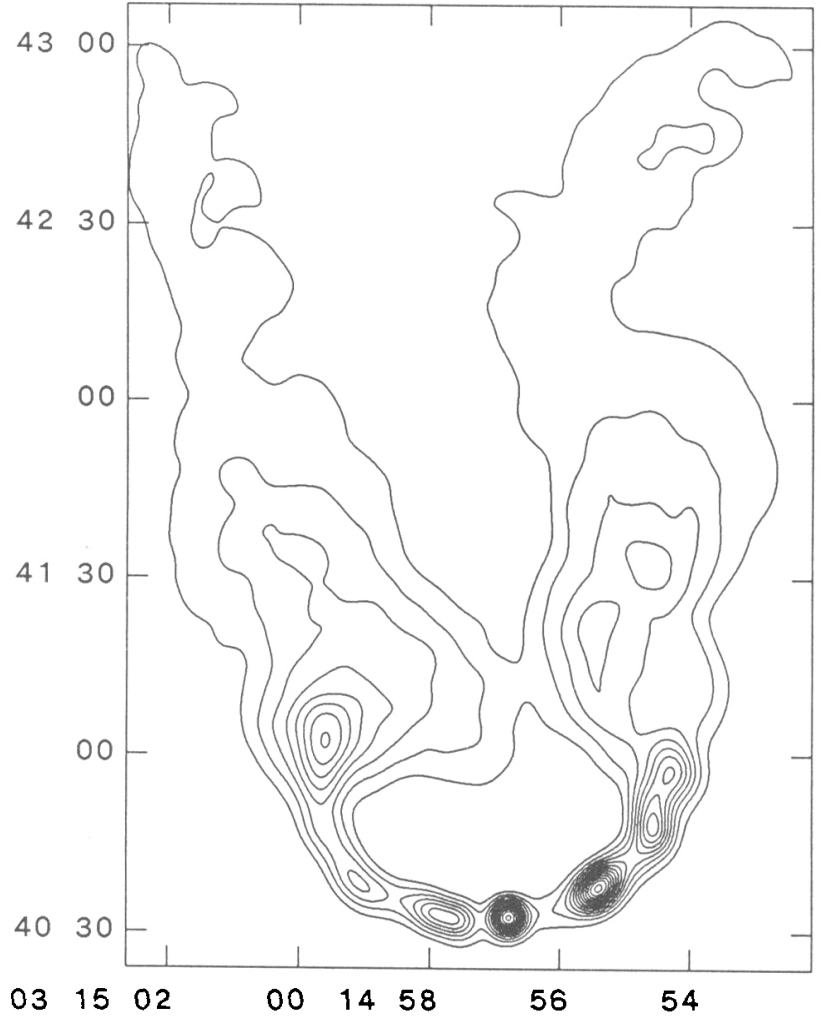

Fig 2 (left). The low-luminosity radio galaxy $3 \mathrm{C} 449$, at $\lambda 22 \mathrm{~cm}$, and $5^{\prime \prime}$ resolution. Contours are at $5 \%$, then at all multiples of $10 \%$ of the maximum.

Fig 3 (right). The head-tail radio galaxy $N G C 1265$, at $\lambda 6 \mathrm{~cm}$, and 5 " resolution. Contours are at multiples of $7.5 \%$ of the maximum of $29 \mathrm{mJy} /$ beam.

Extended regions of extragalactic sources show polarized emission. The distribution of polarized flux is not smooth, and the degree of polarization is generally in the range 10 to $50 \%$, with values up to the theoretical maximum of $\sim 70 \%$ (assuming synchrotron emission, fully ordered magnetic field, and typical spectral index), found near the edges. A striking result comes from the orientation of the projected magnetic field. This is found to be parallel to the source boundaries, or, more generally, perpendicular to strong brightness gradients. An excellent example of this is shown by $3 \mathrm{C} 219$, an intermediate luminosity radio galaxy. The distribution of apparent magnetic field in this source is illustrated in Fig 5.

Thermal gas located either within the emitting volume, or between the source and the Earth, will cause Faraday rotation of the plane of polarization, leading to the possibility that the ionised gas density can be estimated. If the gas is within the emitting volume, the rotation is accompanied by depolarization, but there will be none if the gas is external to the source, unless the observing beam is insufficient to resolve the structure in the external gas. In this situation, an apparent depolarization will result, which can, and has, led to erroneous conclusions concerning internal gas densities. This difficulty, and others, make use of Faraday rotation as a probe into radio source densities difficult [33]. The best current limits come from observing at lower frequencies [34], or by analysing the fluctuations in position angle of polarized flux over the face of the lobe caused by 
randomness in the magnetic field [35]. These techniques give densities $\leq 10^{-5} \mathrm{~cm}^{-3}$. Densities of this order make detection of internal Faraday rotation impossible at frequencies above $1 \mathrm{GHz}$ for typical radio galaxies, so further improvement of these limits will require interferometric polarimetry at lower frequencies.

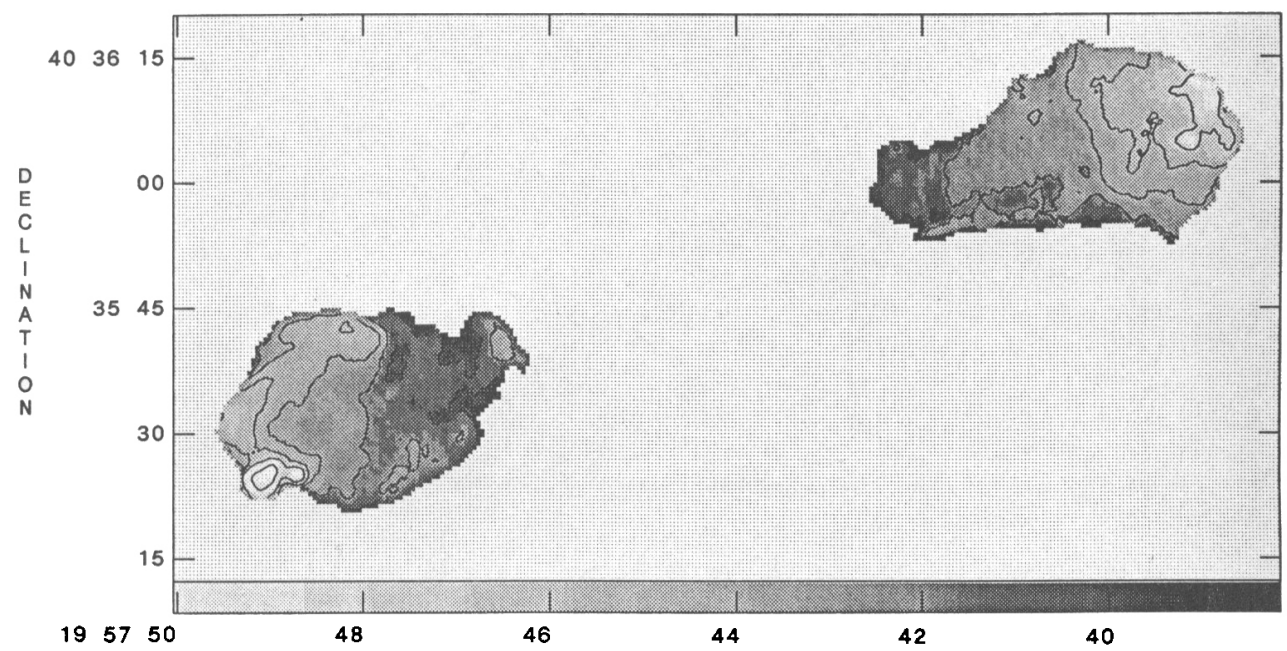

Fig 4. A grey-scale representation of the spectral index between 6 and $20 \mathrm{~cm}$ in Cygnus $A$. The wedge runs from $\alpha=-.55$ (1ight) to -1.8 ( black), so that darker regions have softer (steeper) spectra. The contours are drawn at intervals of 0.2 , from -.6 to -1.6 . Note the flat spectral index in the hot spot regions.

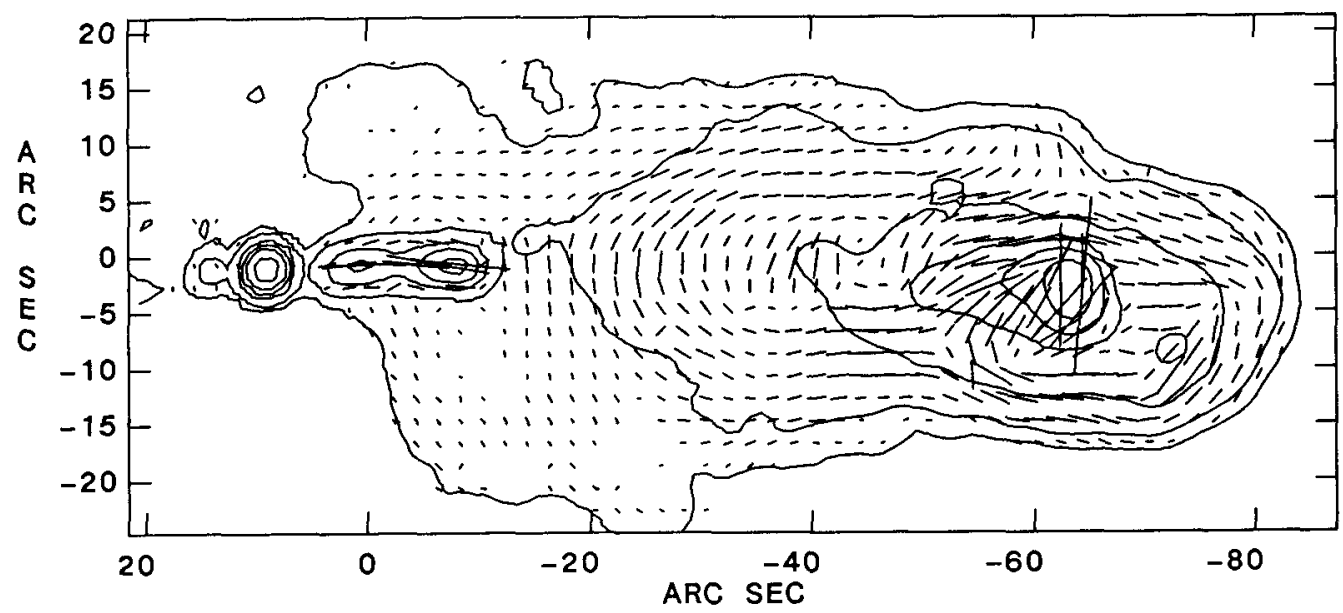

Fig. 5. The projected magnetic field of the SW lobe of 3C219, plotted on a total intensity map at $6 \mathrm{~cm}$ with $2.5^{\prime \prime}$ resolution. The map has been rotated anti-clockwise by 50 degrees. The contours are at $0.5,2,7.5,15,25$, and $50 \%$ of the peak of 53.8 $\mathrm{mJy} / \mathrm{bean}$. The length of each vector is proportional to the polarized $6 \mathrm{~cm}$ brightness at that point, with the maximum vector representing $6.9 \mathrm{mJy} /$ beam. The orientation of the vector gives the direction of the magnetic field.

In general, considerable Faraday rotation, unaccompanied by depolarization, is seen in radio sources. To prove that the rotation is dominated by external gas requires careful imaging polarimetry at a minimum of three frequencies. This has been done for only a few sources, with the result that thermal gas, located within the galaxy, or in a cluster surrounding the galaxy, must be present $[36,37,38]$, and that the internal densities are of order, or less than, the limit given above. 


\section{b) Hot Spots in Extended Sources}

I have previously mentioned that high-luminosity sources contain localized regions of greatly enhanced brightness located at or near the extremities of the source. These regions, known as 'hot spots' in current jargon, are believed to be the termination points of the supersonic beams created by the nuclear engine. Their size is typically a few kiloparsecs, and they contain gradients of $\sim 100 \mathrm{pc}$ scale. Hot spots are probably the sites of strong shocks, and particle acceleration by firstorder Fermi processes is believed to occur $[39,40]$. Momentum deposited by the jet causes the hot spot to advance into the surrounding medium. The cavity thus formed fills with debris spilling out of the hot spot. These structures are of particular importance since they are indicative of the most energetic activity in these extended regions. Recent high resolution VLA maps of Cygnus $A$ at $2 \mathrm{~cm}[41]$ have revealed interesting structure in the SE hotspot, as shown in Fig. 6 . Note the suggestive curved front of emission ahead of the bright raised regions of emission which presumably marks the strong shock terminating the jet flow. Minimum pressures of the relativistic particles and fields are $\sim 10^{-8} \mathrm{dyn} / \mathrm{cm}^{2}$, one to two orders of magnitude greater than in the diffuse lobes. However, due to the small fraction of the source volume these hot spots fill, they contain only $\sim 0.1 \%$ of the total source energy. The lack of spectral curvature in the hotspot emission leads to a replenishment timescale of $\leq 10^{-4}$ years [18], requiring energy inflow in excess of $10^{45} \mathrm{erg} / \mathrm{sec}$. This is clearly a lower bound to the jet kinetic energy flux in this source.

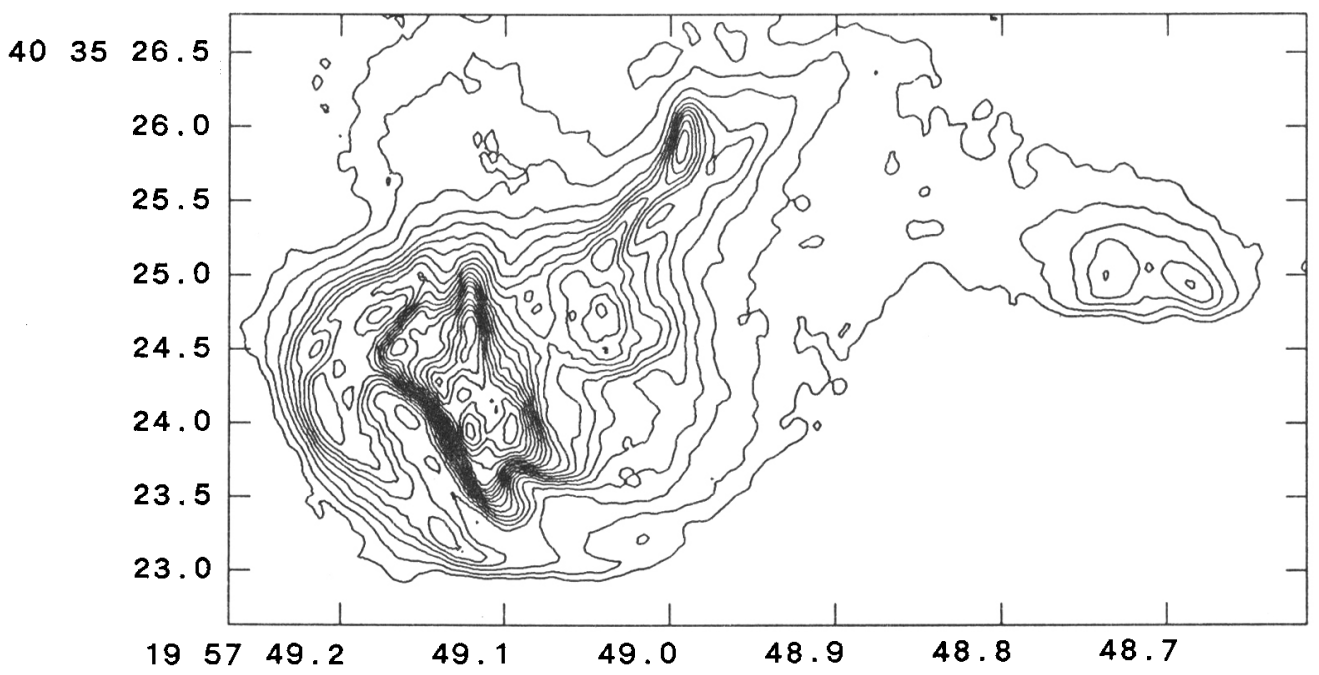

Fig. 6. The SE hot spot of Cygnus $A$ at $2 \mathrm{~cm}$ and $0.11^{\prime \prime}$ resolution. Contours are plotted at all multiples of $5 \%$ of the peak of $120 \mathrm{mJy} / \mathrm{beam}$, plus an additional contour at $2 \%$ of this value. The beam is believed to enter from the NW, and is stopped in a strong shock, which is located at the bright flat-topped region. The interpretation of the curved emission in front of the Mach disk is unclear, it may represent a standoff bow shock. The magnetic field is well aligned with the brightness contours in this region.

Optical observations of hot spots are sparse [1,2]. There is no detection of optical emission from the hot spots of Cygnus A [42]. However, convincing detection of the southern hotspot in $3 \mathrm{C} 33$ has been reported [43], and recently, this emission has been shown to be polarized, and hence, synchrotron in origin [44]. Optical observations of synchrotron emission are important, since they set stringent conditions on particle lifetimes which in turn can be used to set limits on the locations of particle acceleration. 


\section{c) Jets in Extended Sources}

I have already stated that jets are ubiquitous, having been found in all classes of extragalactic radio source, including radio galaxies and quasars, from the most luminous, to the weakest, and probably in some ordinary galaxies as well. Detected jets are always accompanied by core emission - that is, structure on parsec scales or less. There is great variation, however, in the detectability of jets [45]. Prominent jets are common amongst low luminosity radio galaxies and high lumiminosity quasars; detection rates of definite jets in complete samples of both classes of source exceed $50 \%$, and reach $80 \%$ for some galaxy samples and $70 \%$ for some QSR samples. However, amongst high luminosity radio galaxies, the detection rate is $<10 \%$. There is some evidence that the difference is due to the prominence of the core emission that is, there may be correlation between jet flux and core flux [46]. However, clarification of this hypothesis will require considerably larger samples of data than are now available - especially needed, and currently lacking, are accurate measures of integrated jet luminosity.

i) Tota1 Intensity The new imaging instruments have shown us a great range in jet morphologies. To show this range, I display maps of three jets, covering a wide range of source luminosity. Figs. $7 \mathrm{a}, 7 \mathrm{~b}$, and $7 \mathrm{c}$ show the jet in the spectacular, intermediate-luminosity radio source NGC6251. Note that the jet is two-sided, but strongly asymmetric in brightness, with the strong jet leading to a 'warm spot' imbedded in an extensive lobe, and the counter-jet apparently unterminated by any lobe or hot-spot. This is due only to the very large angular size of the source lower resolution maps $[36,47]$ show an extended component, some $20^{\prime}$ in diameter, centered $10^{\prime} \mathrm{S}$ of the Easternmost knot shown in Fig 7a. Also note that the jet shows rotation (or ' $S$ ') symmetry. If due to precession, this places strong upper limits on the jet velocity, completely discordant with the brightness asymmetry being due to relativistic beaming in any ballistic jet model (see [48] for an explanation using $a$ non-ballistic mode1). Fig. $7 \mathrm{~b}$ shows the inner $4^{\prime}$ of the main jet, displaying the knotty structure and small-scale oscillatory structure, and Fig. 7c displays a highresolution map of the inner $50^{\prime \prime}$ of the jet, clearly showing the non-uniform spreading. For details of this source, see [36]. Fig. 8 shows the symmetric jet in NGC1265, an example of a 'NAT', or, narrow-angle tail source. The strong bending is due to the passage of the galaxy through the ICM, this allows constraints on the jet density and velocity to be established, as detailed in Mike Norman's lecture. Fig 9 shows the jet in Cygnus $A$, an extreme example of a high-luminosity radio source.

The spectral index, $\alpha$, in the majority of jets 1 ies between -0.6 and -0.75 , slightly flatter than that of the lobes they presumably feed. Some show steepening along their length, providing evidence for depletion of high energy electrons, presumably by radiation losses. Recent work indicates that the one-sided jets have steeper spectra [21].

There is a clear trend for high luminosity sources to have one-sided jets, and for low-luminosity sources to have two-sided jets. If the ratio separating one-from two-sided jets is taken at 4:1 (a quantity with no significance other than being observationally convenient) measured on the outer $90 \%$ of the jet, then the division occurs at $10^{25}$ watt $/ \mathrm{Hz}$ at $1400 \mathrm{MHz}[49,2]$. Note that all jets are one-sided on sufficiently small physical scales.

Jets show numerous 'wiggles', or bends, examples of which are evident in the preceding figures. Both rotation and reflection symmetries are seen (e.g., NGC6251 is an example of the former, 36449 an example of the latter). In addition, smal1scale wiggles are observed, for example, in NGC6251 [36]. It would appear that any or all of a host of processes, such as galaxy motion, nuclear motion, Kelvin-Helmholtz instabilities, precession, and environmental deflection, will be needed to explain these path changes. 


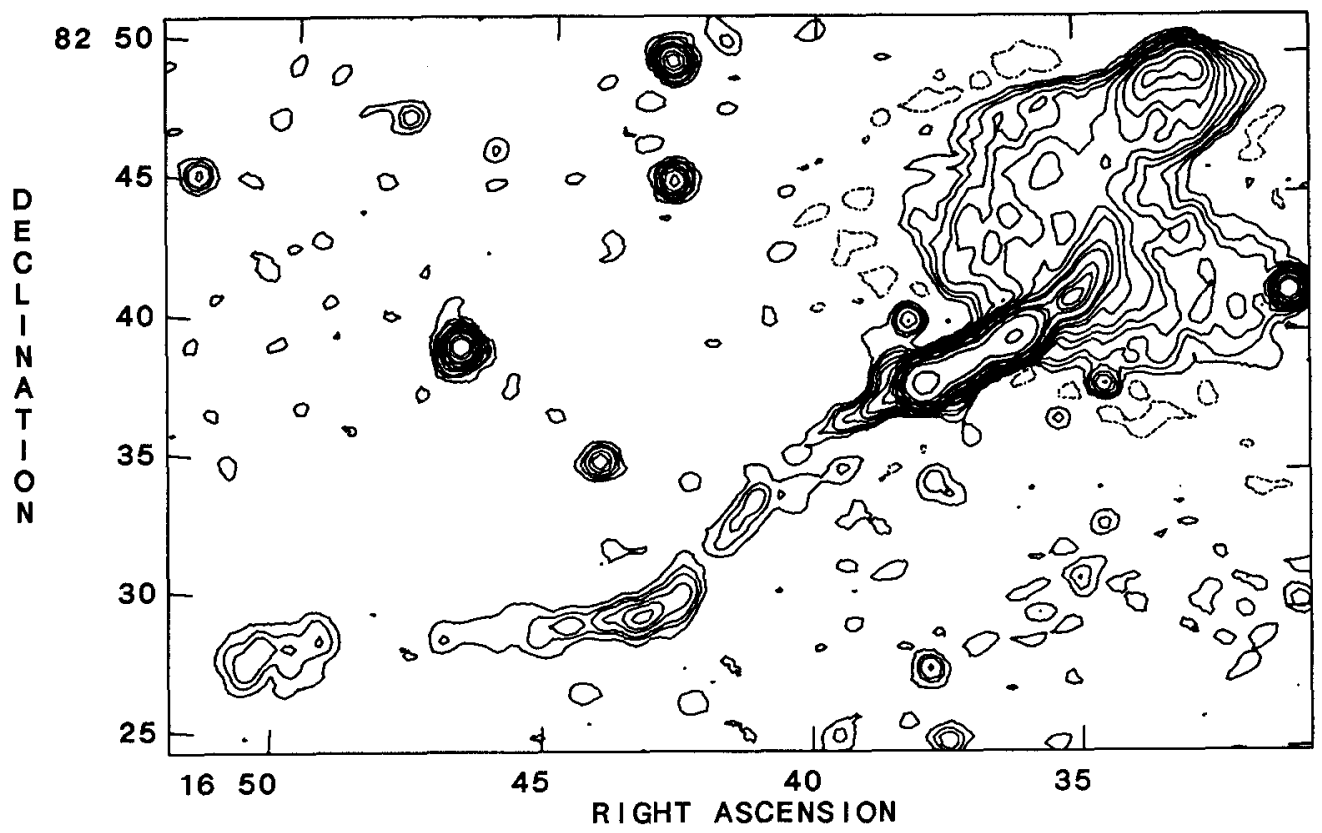

Fig 7a. A map of NGC6251, showing the entire main jet and counter-jet at $20 \mathrm{~cm}$ with 45 arcsec resolution. Contours are at $-1,1,2,3,5,7,10,15,20,30,40,75,100,125$, and 250 times $0.3 \mathrm{mJy} /$ beam, plus a contour at $50 \%$ of the map maximum of $527 \mathrm{mJy} / \mathrm{beam}$ which locates the nucleus and end of the bright inner jet. Note that the jet bends are almost perfectly symmetric under rotation, suggesting precession of the nuclear engine. The western lobe is not visible as it lies far down in the primary antenna power pattern.

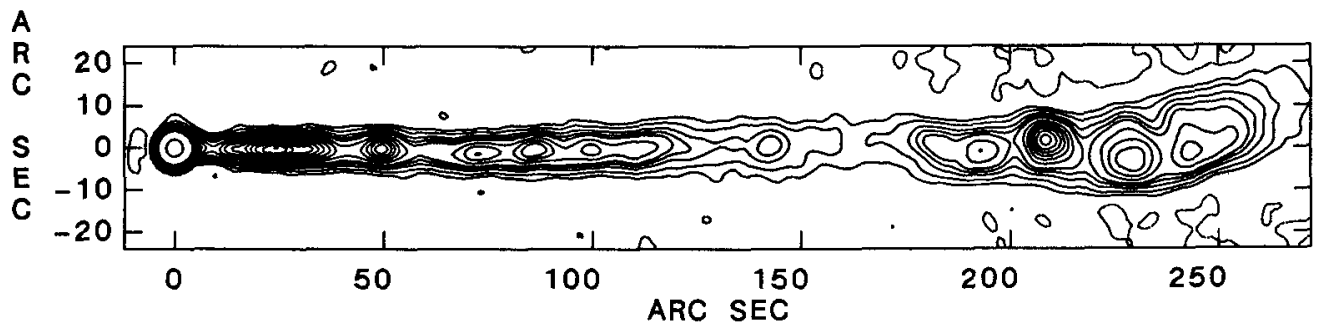

Fig 7b. The main jet of NGC6251, at $22 \mathrm{~cm}$ with $4^{\prime \prime}$ resolution. The map has been rotated clockwise by 26.4 degrees. Contours are at $0.1, .25, .5, .75,1.0,1.5, \ldots, 7.5$ and $50 \%$ of the peak brightness of $424 \mathrm{mJy} /$ beam. Note that the jet is knotty, with significant re-brightnening beyond $175^{\prime \prime}$ from the core. The centroid of the jet oscillates about a fiducial line.

Jets generally spread with increasing distance from the nucleus, although for some, notably 3C219, the jet remains at constant width. The mean spreading rate is clearly inversely correlated with source (or nuclear) luminosity [45, 49], so that highly luminous objects contain slowly spreading jets. In two-sided jets, both sides spread at similar rates, typically within $20 \%$ of each other. For the we11observed jets, the spreading is not linear with distance, but rather operates in distinct stages. There commonly exist 'collimation shoulders', where the local spreading rate is zero. This behavior is clear evidence that the jets are not free, i.e., they do not spread at a constant rate, given by the internal sound speed at the moment they became free. It seems likely that thermal pressure, due to surrounding hot gas, provides the necessary confinement in many, perhaps most, jets, providing that there is approximate equipartition of energy between particles (electrons and 
protons or positrons) and fields. However, in some well-observed sources, notably Virgo A (3C274), the minimum jet pressure in some regions is more than an order of magnitude above any plausible external pressure [50]. If such an overpressure is generally true over the majority of a jet's length, and not due to a local shock (which can raise the pressure by order of the Mach number squared, and is not an equilibrium structure), another confinement mechanism must be found, if the jet is in pressure equilibrium. Self-collimating, current carrying jets have been suggested [51], and the resulting toroidal fields may create an observational 'footprint', if the return current does not lie on the outside of the jet. There is no observational evidence of such fields yet, but the experiment is a difficult one.

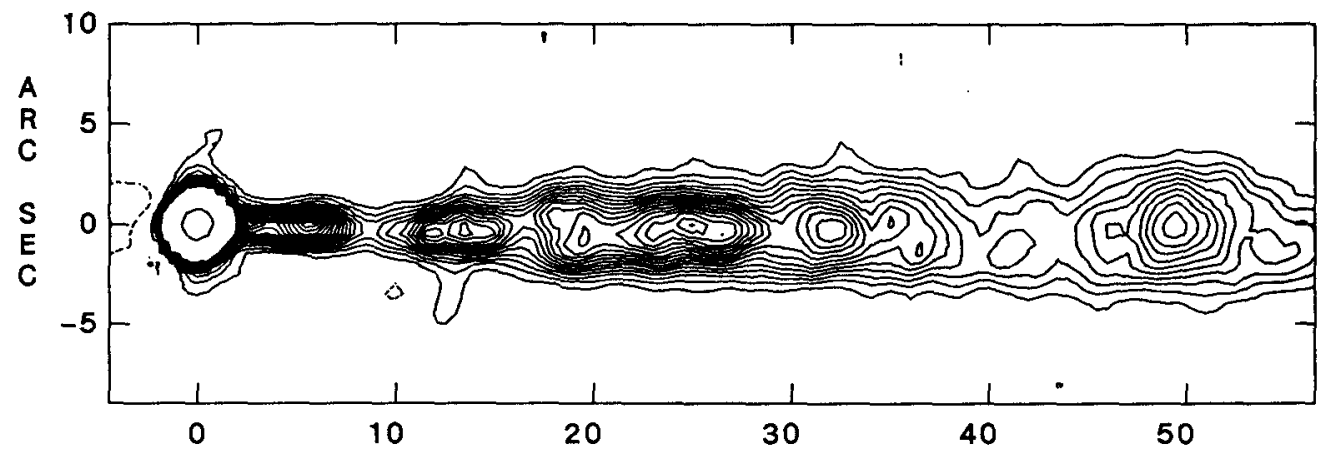

Fig 7c. A high resolution image of the jet in NGC6251 at $18 \mathrm{~cm}$. Contours are at multiples of $.297 \mathrm{mJy} / \mathrm{beam}$, with a contour at $50 \%$ of the peak brightness of 450 mJy/beam to show the resolution of $1.4 "$. The non-monotonic spreading of the jet is clear in this figure.

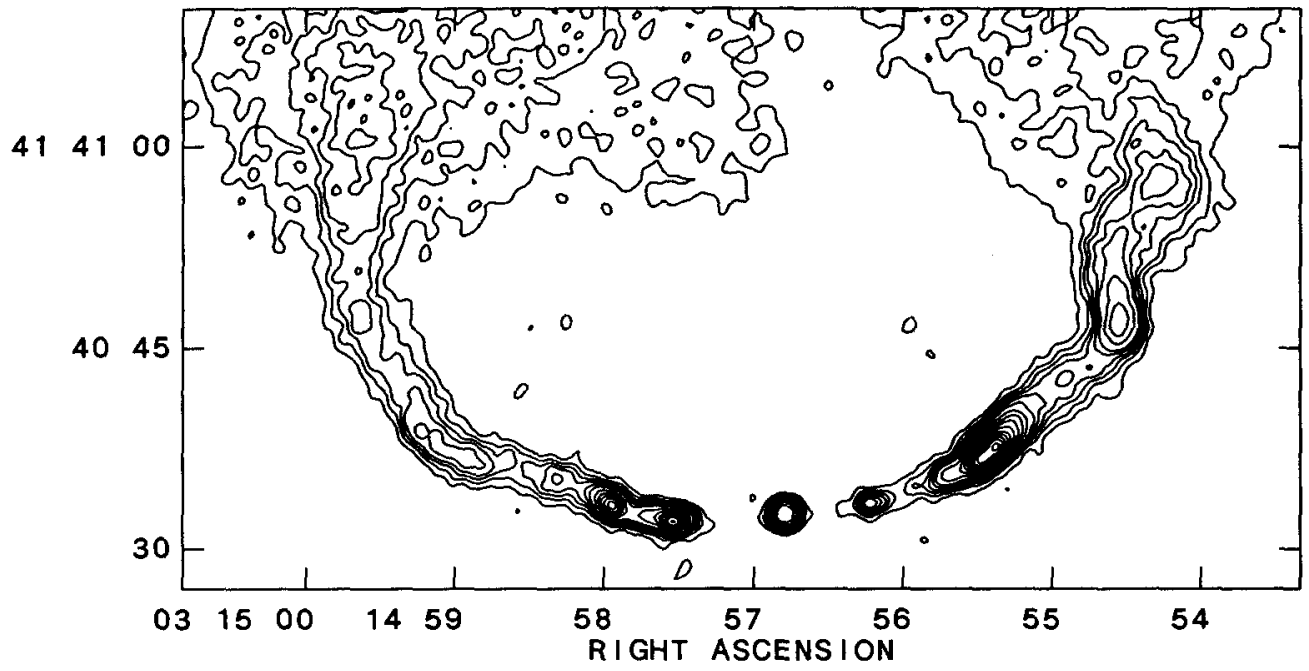

Fig 8. Image of the head-tail radio galaxy NGC1265 at $6 \mathrm{~cm}$ with $0.4^{\prime \prime}$ resolution (?). Contours are drawn at $-.75, .75,1.5,2.25,3,4,5,7, \ldots 30$, and $50 \%$ of the peak brightness of $25 \mathrm{mJy} /$ beam. The general swept-back shape of the jets is convincingly explained by ram-pressure of the galaxy advancing through the ICM. The 'wiggles' in the jet could be caused by fluid instabilities, or by precession of the primary collimator.

The evolution of brightness with length along almost all jets is sub-adiabatic $[2,36,49]$, where 'adiabatic' is here defined as the expected evolution when fields are conserved and particles do work upon expansion down the jet. The implication of this result is that, providing the jet is not decelerating, particle acceleration 
must be continuing along the length of the jet, perhaps due to weak shocks set up by irregularities along the edges of the channel [52], or to MHD turbulence (see [53] for a review of these processes). For jets in low-luminosity sources, sufficient entrainment may occur so that the resultant deceleration will cause enhancement of emission through adiabatic compression [54]. The jets in this model are of low velocity and are barely supersonic.

D

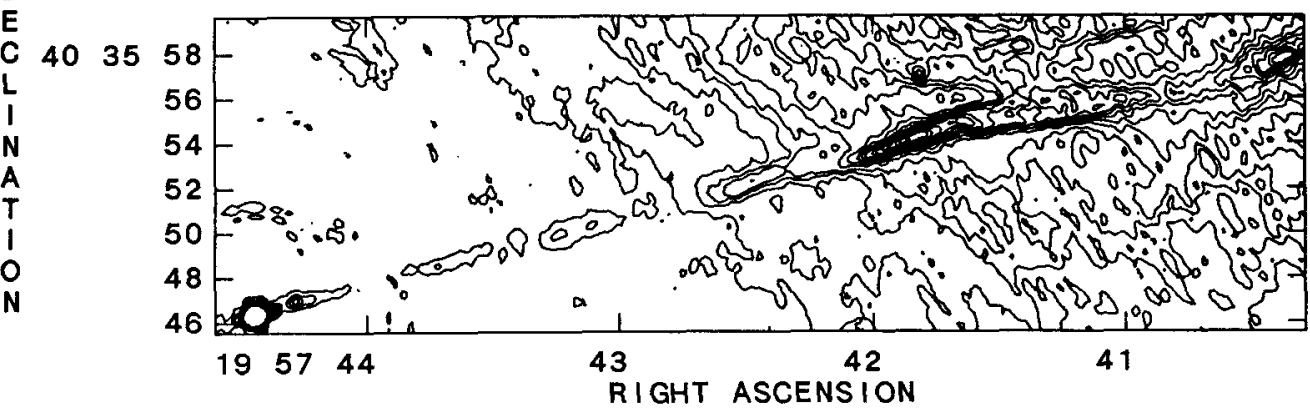

Fig 9. The jet in the luminous radio galaxy Cygnus $A$ at $6 \mathrm{~cm}$ with $0.35^{\prime \prime}$ resolution. Contours are at $1,2,3,4,6,8, \ldots, 22$ times $3.2 \mathrm{Jy} / \mathrm{beam}$. As in other high-luminosity sources, the jet is one-sided, and has a longitudinal magnetic field configuration.

ii) Polarisation Jet emission is commonly linearly polarised, often highly so. As with the extended lobes, the degree of polarization is often highest at the edges this is especially noted on the edges of the jet is B-parallel regions, and along the outside of bends, leading to an interpretation in terms of shearing [55]. Bperpendicular jets are generally most highly polarised in the center. The minimum in polarised emission in the center regions of some jets can be interpreted as a superposition of parallel and perpendicular components along the line of sight. I have already noted that observations of the degree of polarization as a function of frequency can give information on the internal density of thermal matter, and have noted that these measurements are difficult in practice. The same caveats apply here to jets. The only safe result so far is that there is no depolarisation seen in any jet, and limits on the internal density are in the order $\leq 10^{-3} \mathrm{~cm}^{-3}$ [36].

Radio polarimetry shows that the apparent magnetic field highly ordered on the large scale (this does not rule out small-scale turbulence, since shearing of this turbulence will allow high degrees of polarization [55]). The orientation of the field in almost all cases is aligned either parallel or perpendicular to the axis of the jet [49]. The significant exception to this rule occurs in the more extreme brightness enhancements, or knots, visible in many jets. Field directions in this situation can be oblique, and are interpreted in terms of shocks [36,50]. The presence of parallel or perpendicular field is very strongly correlated with source luminosity. Sources with $20-\mathrm{cm}$ spectral luminosity exceeding $\sim 3 \times 10^{24} \mathrm{Watt} / \mathrm{Hz}$ invariably have jets with purely longitudinal fields. Because of the previously noted correlation between sidedness and luminosity, another way of stating this is that purely one-sided jets have longitudinal fields. Sources weaker than this power have predominantly parallel fields, although the brighter side will often initially have a parallel field regime which then flips to a perpendicular one. An important exception to the above correlations occurs along the edges of jets, especially where bends occur. Under this circumstance, the field is usually parallel to the brightness contours; this strongly suggests shearing is important in determining the mean magnetic field alignment. Examples of these trends are given in Figs. 10 through 13, which show the projected fields of $3 C 449,3 \mathrm{C} 219,0326+396$, and NGC 1265 .

iii) VLB Observations A number of extended sources with strong core emission have been mapped with VLB techniques. Observations of 4 very extended radio galaxies [56] showed the cores to be extended and closely aligned with the large scale structure. Similar results from a sample of extended quasars [57] have also been 
reported. In Fig 13 is reproduced the VLB jet in NGC6251 [48]. This jet is one-sided to a brightness ratio of $80: 1$, and points to within 4 degrees of the arcsecond jet. Most of the bend occurs within 15 mas of the core - the knot at the end is closely aligned with the arcsecond structure. This result impressively demonstrates the stability of the nuclear engine, since the large scale structure, in some sources, probably exceeds $10^{9} \mathrm{yr}$ in age. In all cases, the milliarcsecond structure is onesided, and on the same side as the large-scale jet, if one is known, although in 3C390.3, different observers have found the jet on opposite sides of the core $[56,58]$.

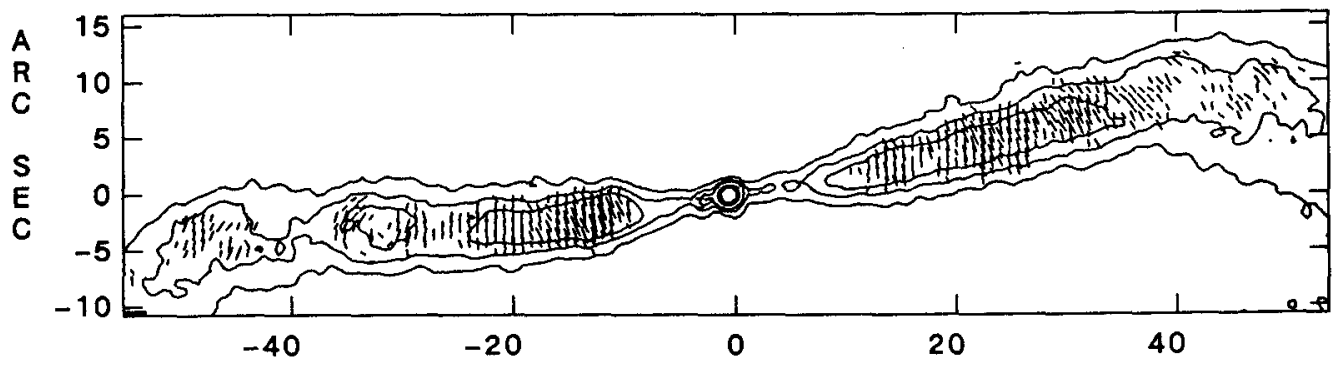

Fig. 10. Magnetic field direction in 3C449, derived by fitting the polarised flux position angle versus $\lambda^{2}$, and solving for the zero wavelength intercept. The contours plotted are from a $22 \mathrm{~cm}$ map at $1.3^{\prime \prime}$ resolution, and are at levels of $1.5,5$, 10,30 , and $50 \%$ of the peak flux of $17 \mathrm{mJy} / \mathrm{beam}$. The lengths of the vectors are proportional to the polarized flux brightness at $6 \mathrm{~cm}$, the orientation is that of the projected magnetic field. Note that the field is closely transverse to the jet.
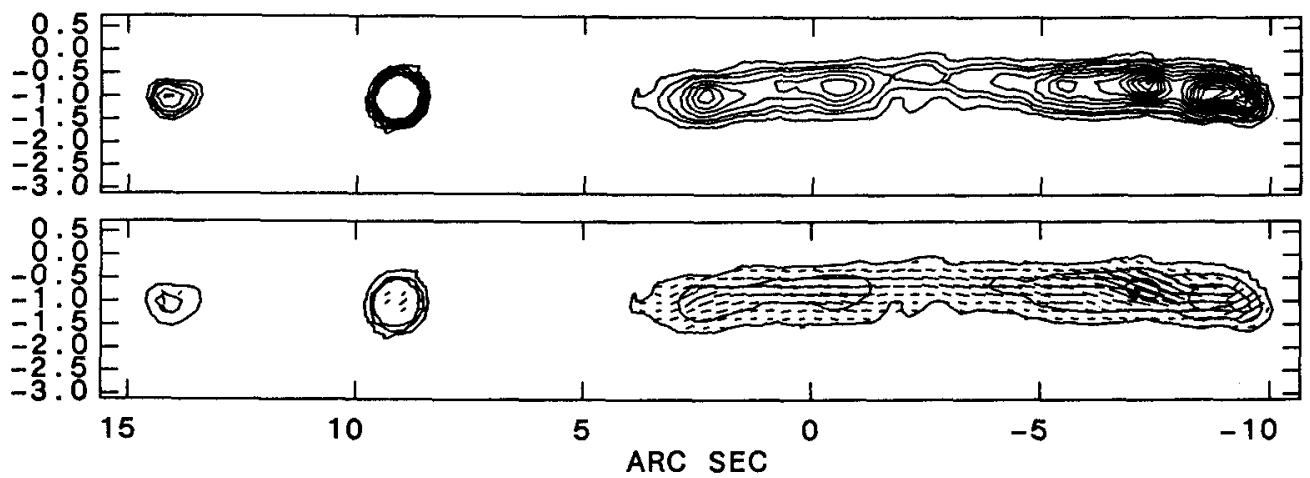

Fig. 11. The jet in $3 \mathrm{C} 219$ at $6 \mathrm{~cm}$ with $0.4^{\prime \prime}$ resolution (above), and the apparent magnetic field (below), derived by rotating the observed $6-\mathrm{cm}$ electric vectors by 90 degrees, a valid procedure since the rotation measure is less than 50 radians per sq. meter. The vector lengths are proportional to polarised brightness. Contour levels are at $.4, .7,1,1.5, \ldots 5.5 \%$ of the peak brightness of $43 \mathrm{mJy} / \mathrm{beam}$ (above), and $0.4,1.5$, and $4 \%$ of the same peak (below).

iv) Other Bands Few jets have been detected in other wavebands. The jet in M87 (Virgo A) has been clearly detected in both infra-red and optical $[59,60]$. Its structure in both bands exactly parallels the radio [50] and there is little doubt that the emission mechanism is synchrotron. In $3 \mathrm{C} 273$, the optically detected jet [61] is clearly related to the radio, but here the differences are striking, as the optical jet and radio jet maxima are not coincident. There are significant differences in polarization as well [62]. Other optically detected jets, less well studied, have been found in 3C31, 3C66B [63], and, tentatively, 0521-36, as reported at this Colloquium by Sol and Cayette. In all the above observations, the emission is a featureless continuum, and is coincident with the radio emission. 

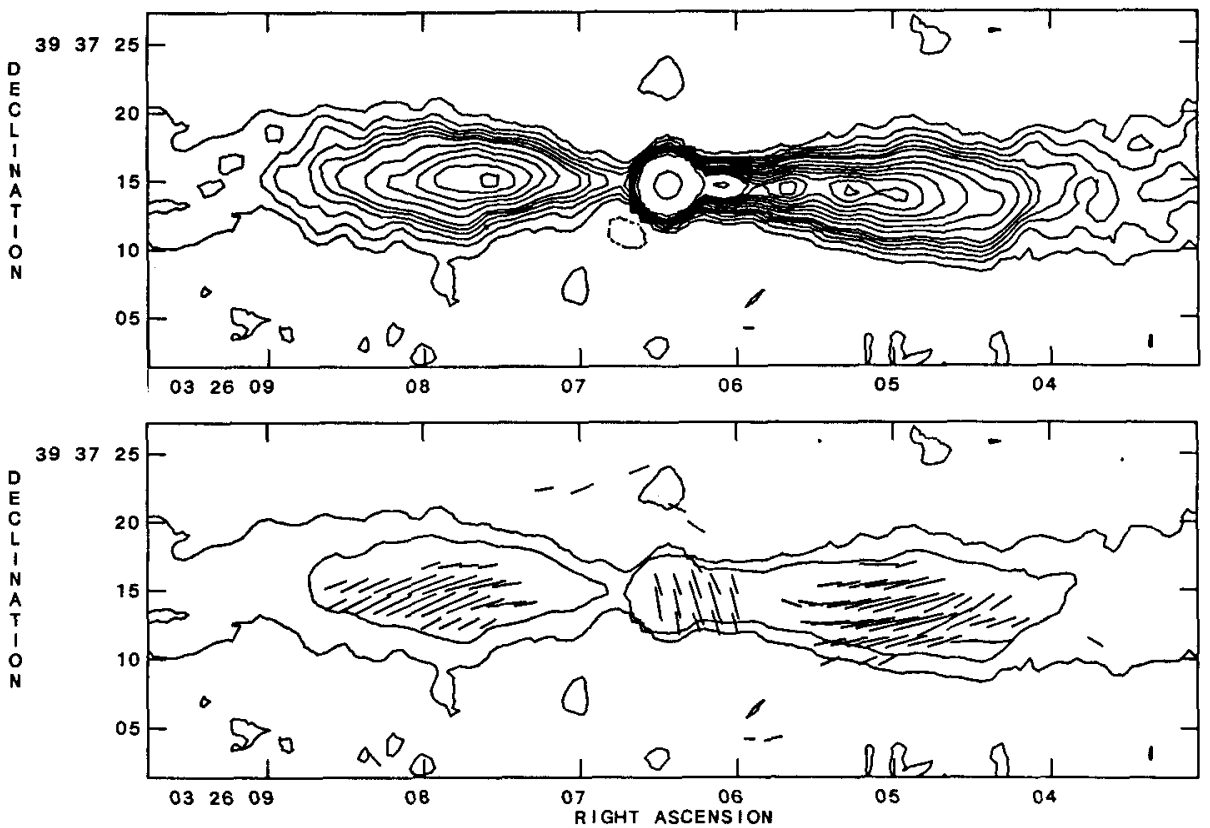

Fig. 12. Maps of 0326+396, a low-1uminosity radio galaxy, at $6 \mathrm{~cm}$. (Above), the total intensity: The jet is symmetric on scales greater than $\sim 10 "$, but one-sided on scales less than $\sim 5^{\prime \prime}$. Contour levels at $-1,1,2,3,4,5,6,8, \ldots .20,30 \mathrm{x} .01 \mathrm{mJy} / \mathrm{beam}$, with a contour at $50 \%$ of the peak brightness of $76 \mathrm{mJy} / \mathrm{beam}$. (Below) The apparent Evectors, the projected B-vectors will be nearly orthogonal to these. Note that the symmetric parts of the jet have perpendicular fields, but the one-sided base of the Western jet has a longitudinal (parallel) field.

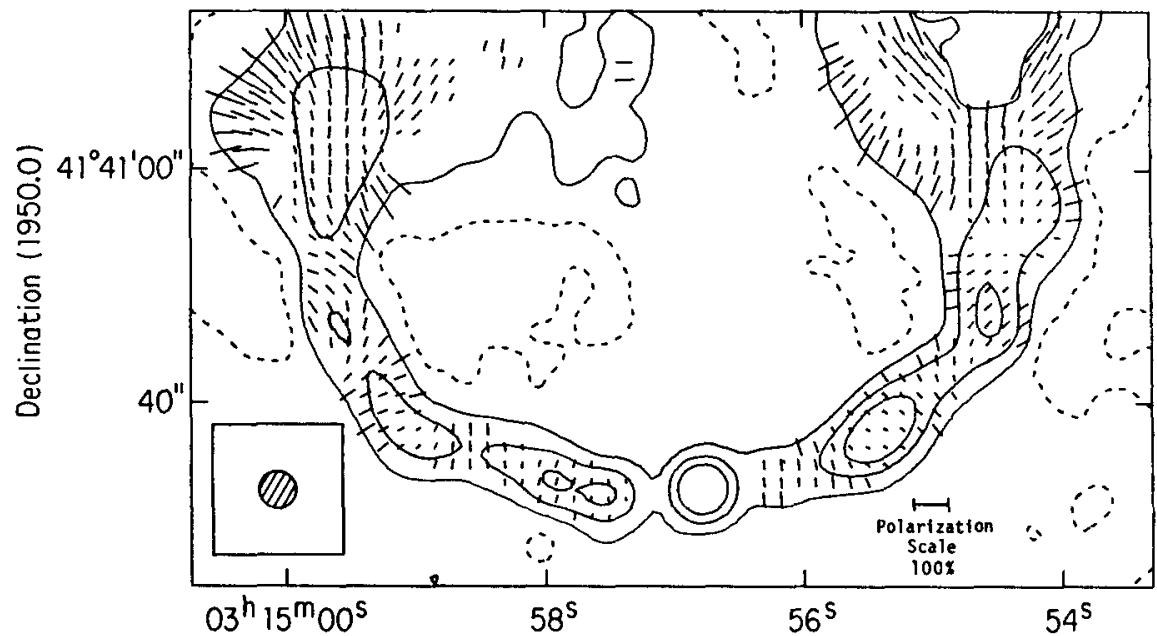

Fig. 13. The position angle of the electric field for the inner jets of NGC1265. The projected B-field will be perpendicular to the vectors. Note that the field is longitudinal throughout the curved region, unlike other two-sided, straight jets. This configuration is probably due to strong shearing caused by ram-pressure bending of the jets. After the bends, the field becomes transverse in the center of the jets, but remains parallel along the edges. 


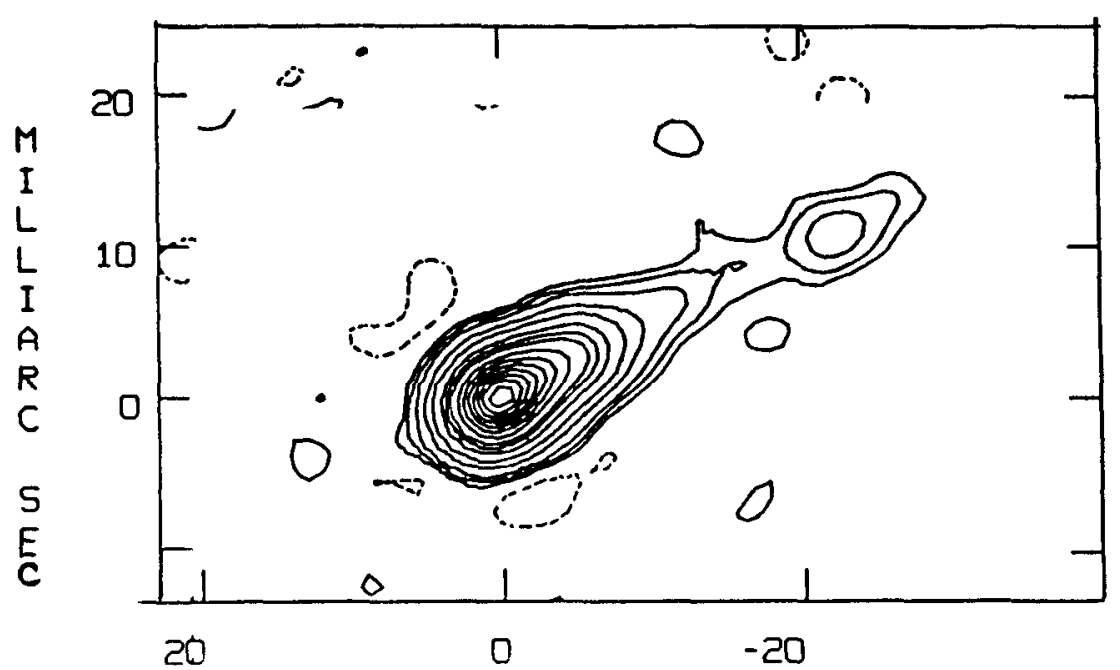

Fig. 14. The milliarcsecond jet in NGC6251 at $18 \mathrm{~cm}$ with $3 \mathrm{milliarcsecond} \mathrm{resolution,}$ from $[48]$.

Eighteen radio galaxies and quasars show extended optical line emission [64]. This emission is not coincident with radio features, but lies near, and slightly outside, bends in the jets or near source boundaries, but still within the optical galaxies, where gas densities can be expected to be high. The luminosity in the emission lines can exceed the total radio luminosity.

The line emission is interpreted in terms of photoionization by a synchrotron powerlaw continuum, or by shock heating. In either case, the required heating is believed to come from the jet kinetic energy flux. Six of these objects have detailed observations which are summarized in [64]. The Doppler shifts seen in these data are believed too small to supply the required energy, and are thought to represent motion in the heated gas outside the jet flow.

$\mathrm{X}$-ray observations from radio jets have been recently reviewed [65]. Only three jet radio sources are known $X$-ray sources: $M 87$ [66], where the identification with synchrotron emission from the jet is secure; 3C273 [67], where other emission processes are possible, but unlikely [65], and Cen A [68]. In addition, $\gamma$-rays are found from the region of 36273 [69], but their origin remains uncertain.

\section{2) COMPACT SOURCES}

Prior to the era of high-dynamic range imaging, it was widely perceived that there were two distinct classes of sources. The first, extended sources, have been described above. The second, core-dominated, or compact, sources, are the subject of this section. The basic morphological characteristics of these objects are: The flux density is dominated by a single, unresolved (on the arcsecond scale), usually flat-spectrum source, most commonly identified with a quasar or BL Lac object. The radio emission is normally variable, especially at shorter radio wavelengths. Until recently, virtually none of these objects was known to possess structure on the arcsecond scale. Better imaging has now revealed that at least $50 \%$ of these objects contain extended stuctures on this scale, often in the form of a 'jet-1ike' extension, usually one-sided $[70,71,72]$. Few sources have emission on both sides of the core, and the flux-density ratio between components so located is typically much higher than in extended radio sources. Recently, some of these objects have been found to possess large-scale extended structure [73]. The polarization characteristics of the jet-like emission follows the guidelines for extended sources. All elongated structures have longitudinal fields, and degrees of polarization typically 10 to $30 \%$. No source shows evidence of depolarization, and for 30273 , the limit on jet thermal density is $\leq 10^{-4} \mathrm{~cm}^{-3}$. 
I illustrate these remarks in Fig. 15 and 16 , showing the arcsecond emission from the well-known quasars $3 \mathrm{C} 273$ and $3 \mathrm{C} 345$. The former is noteworthy since there is no trace of a counter-jet (to $~ 500: 1$ ratio), or extended halo. By way of contrast, 3C345 is imbedded in a large and diffuse halo [73].

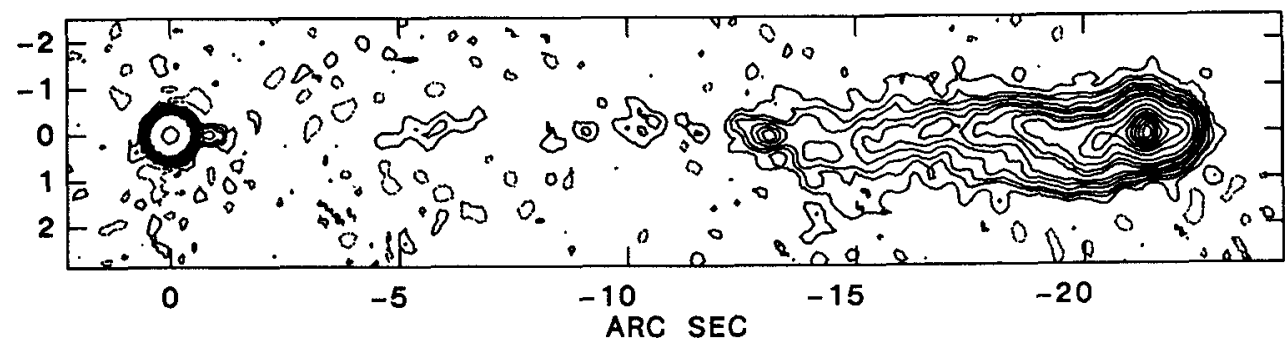

Fig. 15. The arcsecond jet in $3 \mathrm{C} 273$ at $6 \mathrm{~cm}$ with $0.35^{\prime \prime}$ resolution, rotated clockwise by 137.7 degrees. Contour levels at $-1,1,2,3,5,7.5,10,15,20,30,50,75,100, \ldots, 200 \mathrm{x}$ $2.45 \mathrm{mJy} /$ beam, plus one contour at $50 \%$ of the peak of $32.7 \mathrm{Jy} /$ beam .

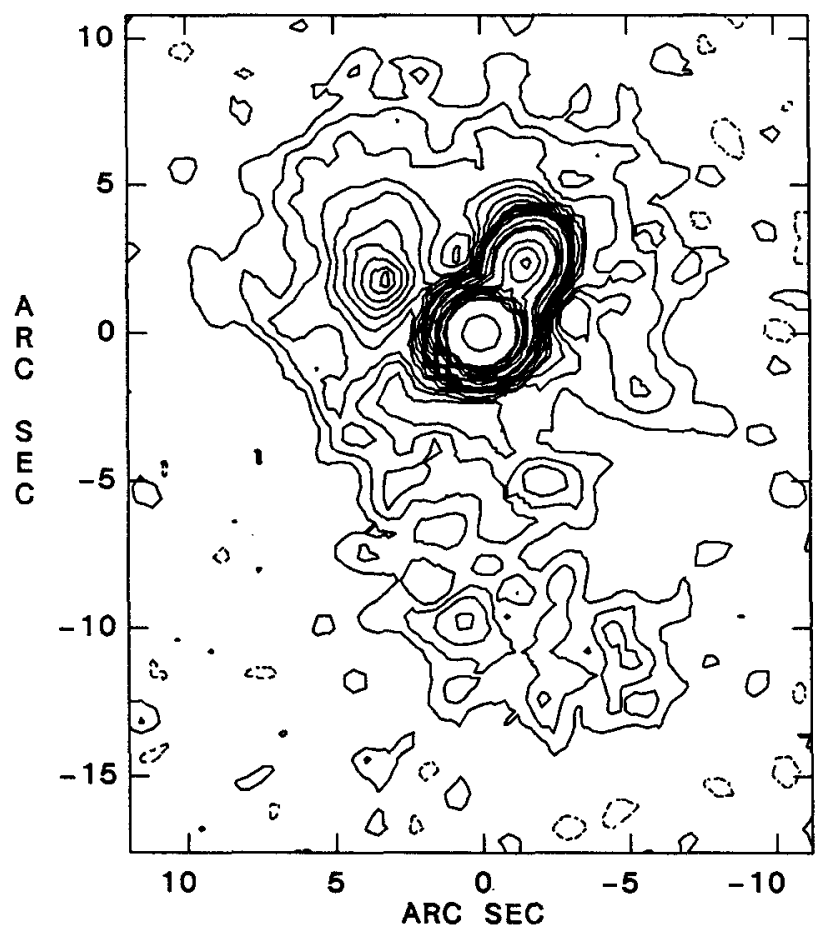

Fig. 16. The arcsecond structure of $3 \mathrm{C} 345$ at $20 \mathrm{~cm}$, with $1.4^{\text {" }}$ resolution. Contours are $-1,1,2,3,5,7,10, \ldots, 21,25,30,50,75,100,200, \ldots, 500,750,1000$ and $5000 \times 0.85$ mJy/beam. The last contour delineates the half-power beam. Note the extensive diffuse emission surrounding the inverted-spectrum core (at 0,0$)$ and one-sided jet (in p.a. - 30).

On the milliarcsecond scale, VLB observations show the majority of compact sources are still dominated by an unresolved, inverted-spectrum core, but that in addition there is usually a one-sided, optically thin structure, commonly jet-1ike [58]. These jets are often highly curved, occasionally exceeding 90 degrees. The small angular size of the core implies high brightness, which in turn implies inverse Compton scattering of radio and infra-red photons to X-ray energies. In most cases, the observed X-ray fluxes 1 ie well below that expected from the radio brightness. 
Consequences of this will be discussed later. Little data are available on the polarization of the jet components - VLB polarimetry is still in its infancy [74]. Recent results show generally longitudinal fields, but in 3C345, one knot has transverse field [75]. Integrated polarizations of the cores show the position angles of polarized flux are highly correlated with the VLB structure, in the sense that the projected magnetic fields are parallel to the radio jets [76], as would be expected in one-sided jets, with the rules from the large-scale structure [49].

Easily the most significant observational characteristic of this class of objects is 'superluminal' motion - the outward motion (from the core) which, when combined with a distance estimate, generally from the red-shift, implies jet velocities greater than light. The discovery of this effect [77] stimulated many theories, reviewed in $[78,79]$. Since the advent of closure mapping [80,81], detailed maps (rather than model fits) made using up to 16 antennas are available, with the result that there is no doubt about the reality of the effect. There are now 9 known superluminal sources $[82,83]$, and it is likely that a significant fraction of all compact sources will prove to have this characteristic, although current estimates of this fraction are difficult to make [84].

The apparent velocities range from just over $c$ to $v / c \sim 8$ (for $H_{0}=100$ ), although it must be emphasized that not all VLBI sources show expansion. Some, with core-jet, or simple double morphologies, are apparently stable. Proper motion studies require distinct features for tracking, and those objects with smooth jets, as in M87, may well be moving, but with no possibility of being tracked. The simple double morphologies are more puzzling, since they are somewhat of a misfit in current thinking [85]. It must be emphasized that one commonly associates the jet speed with the motion of the knots - but this velocity is quite possibly that of a shock or feature, not of the true flow speed. Only in 36345 can it be definitely stated that the optically thin knots ('jet') are moving away from the optically thick core [86], however, it seems quite unlikely that the converse is physically realistic in any other source. In numerous cases, the motion of individual knots, each ejected at different times, is along different position angles although it is unclear whether the motion is radial or not, or even whether it is rectilinear $[87,88,89]$.

Perhaps the best example of all characteristics mentioned above is the superluminal source $3 \mathrm{C} 120$. This irregular galaxy contains jet-like structure on all scales ranging from milliarcseconds to arcminutes, and has been the object of a longrunning series of observations involving many instruments [90]. In Fig. 17 is displayed a montage, illustrating the remarkable continuity of jet structure. Note the persistent asymmetry in the linear (jet) features, and the more symmetric largescale emission evident in the largest scale structure.

of the many models, by far the most widely accepted is that employing a relativistic jet moving close to the line of sight [91]. This model is a special case of the more general relativistic spherical expansion suggested by REES [92] to account for brightness temperatures exceeding the $10^{12}$ 'Compton limit' implied by rapid time variability of the nuclear flux density.

Under this situation, the apparent trasverse velocity will be greatly accelerated, and relativistic effects cause the emission to be 'beamed' into a narrow forward cone, with the result that the 'on-axis' intensity will be greately enhanced. This simple, kinematic model convincingly explains all the major observational characteristics of compact radio sources, including: superluminal motion, very high radio brightness of the core, high curvature of the jets, lack of inverse-Compton $X-$ rays, rapid variability, and one-sided jet structure. In addition, it seems natural that these jets will evolve into the large-scale structures which feed the lobes of the extended sources, although there are difficulties in disposing of waste heat in retarding a relativistic beam. Application of this model to the data from superluminal sources results in bulk Lorentz factors, $\gamma$, of 5 to 20 , and angles of propagation, from the line of sight, of $\leq 7$ degrees. This is not to say that all peculiarities found by observers are comfortably explained by this model -there is still plenty of room for observations and theoretical ideas to compete. 

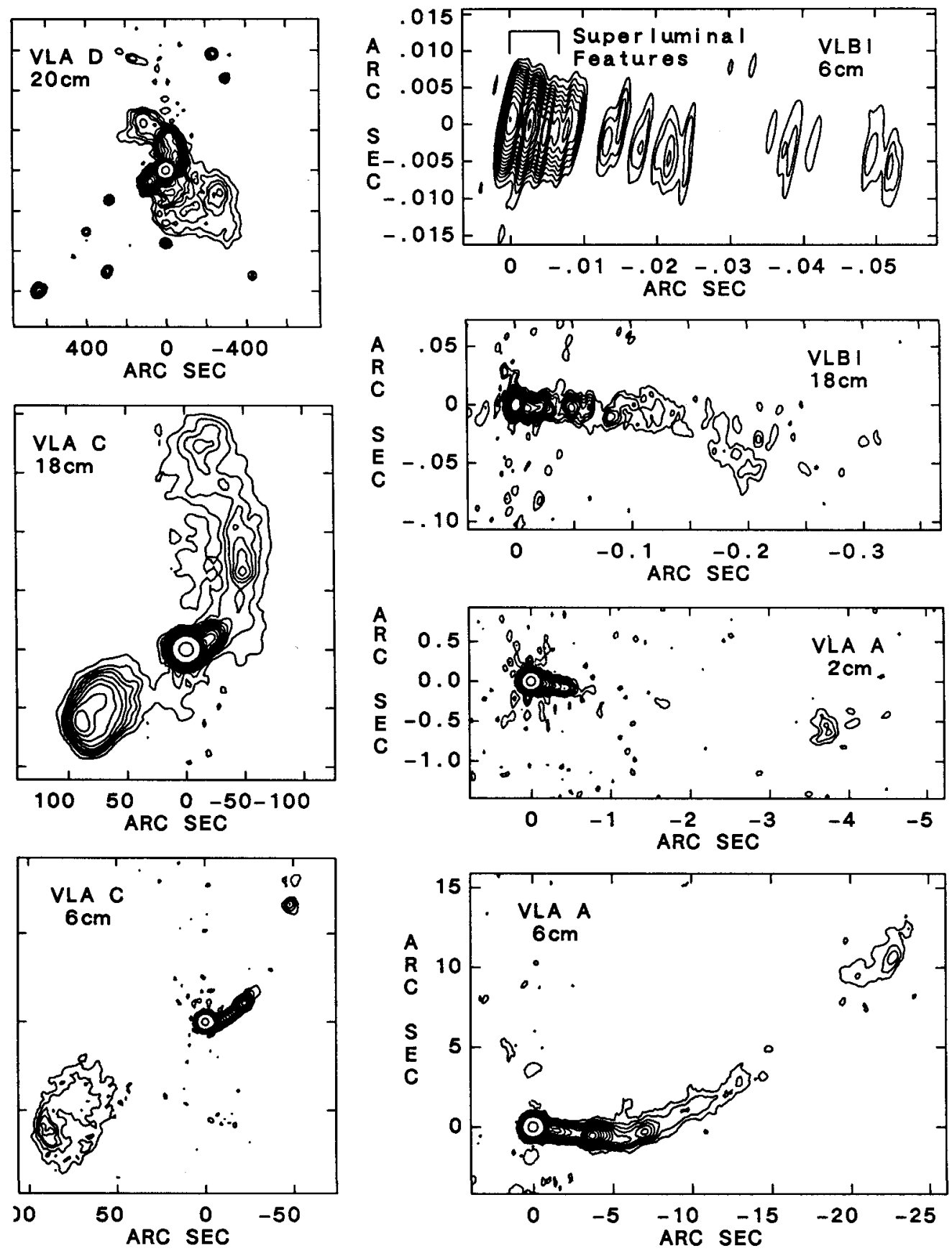

Fig. 17. The radio structure of 3C120. The resolution in the various maps increases going clockwise, starting with $\sim 2$ milliarcseconds in the upper right, and ending with 45 arcsecond in the upper left. All maps of been contoured logarithmically, with yy contours per decade in brightness. 
One important consequence of the relativistic jet model deserves special mention here. If the compact radio sources are all due to relativistic jets pointed close to the line of sight, then the natural question is, what do unbeamed sources look like? Due to the beaming effect, there should be $\sim \gamma^{2}$ unbeamed objects for every beamed one. Since there are hundreds of core- dominated sources, there could be tens of thousands of unbeamed ones, constituting a group commonly known as the 'parent population'. Are the members of this group the 'radio-quiet' quasars, [93], or are they the classical radio doubles [94]. There is considerable interest in the latter suggestion, often called the 'unified scheme', since, as reported above, the majority of compact sources are connected to extended, large-scale, and presumably unbeamed, structure. Observations of Blazars (=BLLacs plus OVVs) [95] have tended to support this scheme, which here identifies the parent population with weak emission from elliptical galaxies. However, optical observations indicate differences in (presumably unbeamed) line emission characteristics between lobe-dominated sources and core-dominated sources [96].

\section{IV) COMMENTS ON VELOCITY AND DENSITY}

Although this is an observational talk, with the two following talks concerning interpretation and theory, some comments are in order, as they represent an observer's perspective. Probably the most important important parameters of astrophysical jets which must be measured are the density and velocity. Unfortunately, it seems clear that the physics of these jets precludes any easy determination of these quantities. The only secure and model-free estimator of velocity is measurement of Doppler shifts, and since the emission process is likely to be non-thermal synchrotron, there appears to be little chance of success in measuring velocities in this way. The next most secure method lies in proper motions; however, here the distance and angle to the line of sight of the motion are required. Furthermore, the technique cannot be applied to large-scale jets, since they contain no features small enough to be followed with sufficient resolution (however, see [97] for another possibility). For density, the situation is nearly as bad, and certainly more frustrating for, as earlier mentioned, although measurement of depolarization enables, in theory, an estimate of internal density, in practice, the topological complexity of the internal fields makes the technique difficult. Thus, we rely upon indirect estimators, which necessarily implies model dependence. The question of jet velocity is of such importance, however, in the physics of jets, that some review of the evidence is in order. This has already been done $[2,45]$, and I give only a cursory recap. Due to all the uncertainties in measurement, it seems prudent to restrict discussion to whether jets are relativistic on various scales.

a) On parsec scales, there seems to be little doubt that relativistic velocities exist. I have given above the major arguments, which center on how easily motions of this type explain the unusual characteristics of compact radio sources. No other model can so easily explain all these things. Whether relativistic motions exist in the active nuclei of EVERY radio source is another matter, since evidence exists for unchanging structures on these scales, although this should not necessarily be taken as evidence for non-relativistic velocities. Also, existence of two-sided structures on these scales is not necessarily an argument for non-relativistic models, providing curvature is allowed, and only a very few such sources are found - and the observations to date do not conflict with the relativistic model.

b) On kiloparsec scales, there is evidence for both relativistic and nonrelativistic velocities. The most telling argument for relativistic velocities is the spatial continuity between $\mathrm{pc}$ and Kpc structures in strong sources. All superluminal sources known so far have one-sided Kpc jet structure on the same side as the pc structure [2]. Eleven of 16 sources with unknown parsec scale jet velocities have Kpc jets on the same side. For these, if the pc jet is relativistic, than the kpc jet is probably relativistic too. Note however, that in both cases, we cannot rule out intrinsically one-sided jets, and in the latter, one-sided nonrelativistic jets. Another important potential argument for relativistic motions is the observations that luminous radio galaxies and quasars have invariably one-sided jets. Observations of powerful, lobe- dominated sources provide an important test of the relativistic beam hypothesis, since these sources are unlikely to be specially oriented. For objects approximately transverse to the line of sight, the jet/counter-jet ratio must be sma1l. Preliminary work [98] indicates that these 
ratios are too large to be due solely to beaming. However, most of these 1 imits are still small $(<5: 1)$, so better observations are needed. To feed both lobes, and maintain one- sided jets without Doppler beaming requires flip-flop models with carefully regulated timing (so as to keep the hot-spots lit up, while the jet points the other way), or asymmetric environmental effects.

On the other hand, in weaker sources, there can be no doubt that the jet velocities are sub-relativistic. Head-tail sources are two-sided and highly bent, a situation which cannot arise from relativistic motion. Similarly, objects with near-perfect symmetry argue for slow motions. In M84 [38], the observed dust lane tells us the brighter jet points away from the line of sight. In addition, the implied size of some objects, when deprojected from angles required to make them one-sided, becomes uncomfortably larger than objects in which beaming is not suspected [61]. For these objects, the asymmetry in the jet emission is likely to be due to differences in the environment through which the jet propagates.

\section{V) FUTURE EXPERIMENTS}

Although much has been learned about the jet phenomenon over the last few years, there is much which is yet unknown, and which is, or will be, accessible to observations. Presented below is a short list of important observations experiments which, in the author's opinion, will be important in better understanding the jet phenomenon. Some of these must await future instrumentation, and others will require long and careful observating programs.

a) Milliarcsecond Scale - The question of jet continuity from mas to arcsecond scales needs attention. $3 \mathrm{C} 120$ shows this continuity, other sources should be similarly mapped.

evolution.

- The evolution of knots - their motion, intensity, width and spectral

- The polarization of knots - including the polarization evolution.

- Complete sample mapping. This would help to answer the question of how prevalent the superluminal phenomenon is.

- VLB mapping of the cores of large sources. The relativistic jet model can tolerate only a very low fraction of these to show relativistic motion.

\section{2) Arcsecond Scale}

- More high-dynamic range mapping of key compact sources to better delineate the radio characteristics of the parent population.

- More data on jet/counter-jet ratios. Few sources have good ratios (e.g. $>10: 1$ ) on this key parameter.

- The question of confinement - how many good examples of over-pressured jets are there? This requires good X-ray data as well as good radio data.

- Depolarization studies. Few jets have had their Faraday screens mapped well enough for unambiguous statements to be made concerning their depolarization. It is also important to better understand the physics of polarization transport in turbulent media.

- Optical studies of jets and hot spots. These give strong limits on electron lifetimes, and delineate the regions of current activity.

- Sensitive X-ray imaging, to detect both synchrotron X-rays, and perhaps even the radio galaxies themselves (which might be seen as regions of depression in X-ray intensity, if the source is imbedded in a hot intra-cluster gas).

\section{VI) ACKNOWLEDGEMENTS}

It is a pleasure to thank the organisers of this Colloquium for the excellent meeting and the opportunity to take part in it. I am grateful to Dave Murphy and Alan Bridle for useful comments on an earlier draft. Special thanks are due to Alan Bridle and Craig Walker for providing the figures of $0326+396$, and $3 \mathrm{C} 120$, respectively, in advance of publication.

\section{REFERENCES}

1. Miley, G.K., 1980. Ann. Rev. Astron. Astrophys., 18, 165 
2. Bridle, A.H., and Perley, R.A., 1984. Ann. Rev. Astron. Astrophys., 22, 319

3. Kellermann, K.I., and Pauliny-Toth, I.I.K., 1981. Ann. Rev. Astron. Astrophys., $\underline{19}, 373$

4. Begelman, M.C., Blandford, R.D., and Rees, M.J., 1984. Rev. Mod. Phys, 56, 255

5. Physics of Energy Transport in Extragalactic Radio Sources, Proc. NRAO Workshop No. 9, ed. A.H. Bridle and J.A. Eilek, NRAO, Green Bank, W. Va. 1984

6. Proceedings of the C.I.T.A. Workshop on 'Jets from Stars and Galaxies', ed. R.N.Henriksen and T.W.Jones, Can. J. Phys., 1986

7. Schwartz, R.D., 1983. Ann. Rev. Astron. Astrophys., 21, 209

8. Lada, C., 1985. Ann. Rev. Astron. Astrophys., 23, 267

9. Hjellming, R.M., and Johnston, K.J., 1985. Preprint. To appear in 'Radio Stars', (Reidel:Dordrecht). ed. - R.M. Hjellming and D.M. Gibson.

10. Margon, B., 1984. Ann. Rev. Astron. Astrophys., 22, 507

11. Hje1lming, R.M., and Johnston, K.J., 1981. Ap. J. 246, L141

12. Hjellming, R.M., and Johnston, K.J., 1985. In preparation

13. Hjellming, R.M., 1973. Science, 182, 1089

14. Geldzahler, B.J. et al., 1983. Ap. J. 273, L65

15. Johnston, K.J., et a1., 1985. In preparation

16. Fomalont, E.B., Geldzahler, B.J., Hjellming, R.M., and Wade, C.M., 1983. Ap. J., 275,802

17 Fomalont, E.B., private communication

18. Hargrave, P.J., and Ryle, M., 1974. MNRAS 166, 305

19. Longair, M.S., Ryle, M., and Scheuer, P.A.G., 1973. MNRAS, 164, 243

20. Blandford, R.D., and Rees, M.J., 1974. MNRAS, 169, 243

21. Bridle, A.H., private communication

22. Mackay, C.D., 1973. MNRAS, 162,1

23. Longair, M.S., and Riley, J.M., 1979. MNRAS, 188,625

24. Fanaroff, B.L., and Riley, J.M., 1974. MNRAS, 167, 31p

25. Perley, R.A., Dreher, J.W., and Cowan, J.J., 1984. Ap.J. 285, L35

26. Perley, R.A., Willis, A.G., and Scott, J.S., 1979, Nature 281, 487

27. O'Dea, C.P., 1984. Ph.D. Thes is., U. of Mass, Amherst.

28. Wellington, K.J., Miley, G.K., and van der Laan, H., 1973. Nature, 244, 502

29. O'Dea, C.P., 1985. Ap.J., 295, 80, 1985

30. Burbidge, G.R., 1956. Ap.J., 124, 416

31. Pacholczyk, A.G., 1970. Radio Astrophysics, W.H. Freeman, San Francisco

32. Jenkins, C.J., and Scheuer, P.A.G., 1976. MNRAS, 174, 327

33. Laing, R.A., 1984. In ref 5, p90

34. Willis, A.G., Wilson, A.S., and Strom, R.G., 1978. Astr. Ap., 66, L1

35. Myers, S.T., and Spangler, S.R., 1985. Ap. J. 291, 52

36. Perley, R.A., Bridle, A.H., and Willis, A.G., 1984. Ap.J. Supp1., 54, 291

37. Perley, R.A., Dreher, J.W., and Carilli, C., 1985. In preparation

38. Laing, R.A., and Bridle, A.H., 1985. In preparation

39. Bel1, A.R., 1978. MNRAS, 182, 147

40. Blandford, R.D., and Ostriker, J.P., 1978. Ap.J. (Lett.), 221, L29

41. Laing, R.A., Scheuer, P.A.G., and Perley, R.A., 1985. In preparation

42. Kronberg, P., van den Bergh, S., and Button, S., 1977. A.J., 82, 315

43. Simkin, S.M., 1979. Ap. J., 234, 56

44. Meisenheimer, K., and Roser, $\mathrm{H} .-\mathrm{J} .$, 1985. Preprint

45. Bridle, A.H., in ref 6

46. Burns, J.0., in ref $5, \mathrm{p} 25$

47. Willis, A.G., Strom, R.G., Perley, R.A., and Bridle, A.H., 1982. In 'IAU Symposium 97, ed. D.S. Heeschen and C.M. Wade (Dordrecht: Reide1) p141

48. Jones, D.L., et al., 1985. Preprint

49. Bridle, A.H., 1984. A.J. 89,979

50. Biretta, J.A., Owen, F.N., and Hardee, P.E., 1983. Ap.J. Lett., 274, L27

51. Benford, G.B. in ref $5, \mathrm{p} 185$

52. Norman, M.L., Smarr, L.L., and Winkler, K.-H.A., 1984. In 'Numerical Astrophysics:, A Festschrift in Honor of James R. Wilson', ed. R. Bowers J. Centre1la, J. LeBlanc, \& M. LeBlanc. Jones and Bartlett: Boston

53. Eilek, J.A. 1984. In ref 5, p216

54. Bicknel1, G.V. 1985. Preprint

55. Laing, R.A., 1981. Ap.J., 248, 87

56. Linfield, R., 1981 Ap.J., 244, 436

57. Zensus, J.A., and Porcas, R.W., 1984. In IAU Symposium 110, ed. R. Fanti 
K. Kellermann and G. Setti. (Dordrecht: Reidel), p163

58. Pearson, T.J., and Readhead, A.C.S., 1984. In ref 57, p15

59. Nieto, J.-L., and Lelievre, G., 1982. Astro \& Astrophys. 109, 95

60. Smith, R.M., Bickne11, G.V., Hyland, A.R., and Jones, T.J., 1983. Ap.J. 266, 69

61. Lelievre, G., Nieto, J.-L., Horville, D., Renard, L., and Servan, B., 1984. Astron \& Astrophys., 138, 49

62. Roser, H.-J., and Meisenheimer, K., 1985. Preprint

63. Butcher, H.R., van Breugel, W.J.M., and Miley, G.K., 1980. Ap.J., 235, 749

64. van Breugel, W., 1985. Preprint

65. Feigelson, E.D., 1985. Preprint

66. Schreier, E.J., et al., 1982. Ap.J., 261, 42

67. Willingdale, R., 1981. MNRAS 194, 56

68. Feigelson, E.D., et a1., 1981. Ap.J. 251, 31

69. Bignami, G.F., et a1., 1981. Astron. \& Astrophys. 93, 71

70. Perley, R.A., 1982. In ref 47, p175

71. Browne et al.,1982. MNRAS, 198, 673

72. Perley, R.A., Fomalont, E.B., and Johnston, K.J., 1982. Ap.J., 255, L93

73. Schilizzi, R.T., and de Bruyn, A.G., 1983. Nature, 303, 26

74. Roberts, D.H., 1984. In ref $57, \mathrm{p} 35$

75. Wardle, J.F.C., 1985. in ref 6

76. Rusk, R., and Seaquist, E.R., 1985. A.J., 90, 30

77. Cohen, M.H., et al., 1971. Ap.J., 170, 207

78. Blandford, R.D., McKee, C.F., and Rees, M.H., 1977. Nature, 267, 211

79. Marscher, A.P., and Scott, J.S., 1980. PASP, 92, 127

80. Readhead, A.C.S., and Wilkinson, P.N., 1978. AP.J., 223, 25

81. Pearson, T.J., and Readhead, A.C.S., 1984. Ann. Rev. Astr. Astrophy., 22,97

82. Cohen, M.H., and Unwin, S.C., 1984. In ref 57, p95

83. Pearson, T.J., Barthel, P.D., Lawrence, C.R., and Readhead, A.C.S., 1985. Preprint

84. Cohen, M.H., 1985. Preprint. To be published in Proceedings of the Salpeter Symposium 'Highlight of Modern Astrophysics', S. Shapiro and S. Teukolsky, editors

85 Phillips, R.B., and Mute1, R.L., 1982. Astron. \& Astrophys., 106, 21

86. Barte1, N., et a1., 1984. In ref 57, p113

87. Unwin, S.C., et al., 1983. Ap.J. 271, 536

88. Cohen, M.H., et al., 1983. Ap.J., $\underline{269}$, L1

89. Biretta, J.A., et al., 1983. Nature, 306,42

90. Walker, R.C., Benson, J.M., and Unwin, S.C. In preparation

91. Blandford, R.D., and Konig1, A., 1979. Ap.J., 232, 34

92. Rees, M.J., 1967. MNRAS, 135, 345

93. Scheuer, P.A.G., and Readhead, A.C.S., 1979. Nature, 277, 182

94. Orr, M.J.L., and Browne, I.W.A., 1982. MNRAS, 200, 1067

95. Antonucci, R.R.J., and Ulvestad, J.S., 1985. Ap.J., 294, 158

96. Heckman, T.M., 1983. Ap.J.Lett., 271, L5

97. Perley, R.A., 1984. In ref 57, p153

98. Wardle, J.F.C., and Potash, R.A., 1984. In ref 5, p30 


\section{QUESTIONS AND ANSWERS}

1) Blandford

Q. Your rotation measure maps of Cygnus A showed gradients in both lobes parallel to the overall source axis. If toroidal magnetic field is responsible for jet collimation, then one would expect to see gradients perpendicular to the source axis. Do you know of any good examples of this phenomenon?

A. None so far. Simple calculations indicate a rotation measure difference of perhaps $20 \mathrm{rad} / \mathrm{m}^{2}$ from one side of a jet to the other, which would mean about a radian turn in the apparent E-vector at $20 \mathrm{~cm}$, a measureable effect. However, there must be polarized emission from behind the jet for the effect to be seen. Thus, a jet in a bright lobe is necessary. There are a few sources which are attractive candidates, but the necessary data has not yet been taken. Proposals to do so have been submitted.

\section{2) Nordlund}

Q. Concerning the 'stepwise' changes of opening angles along some jets (or rather jet-paths or knot-paths). We are seeing a shapshot, so we don't necessarily have to assume that the 'upstream' section of the jet will be re-collimated further downstream. We could be seeing sections of the jet propagating in the wake (channel) blown by the previous jet section, each section wider than the previous one. Only after the medium has a chance to relax would a new narrower section be formed.

A. This independent blob, or plasmon, picture could certainly be occuring if the jet mechanism is discontinuous. However, many of the jets showing this stepwise spreading are quite continuous in appearance. Also, the collapse time of the channel will be quite slow, somewhat longer than the sound crossing time, which for these jets is of order a million years, considerably longer than the time-scale of activity in the nuclear core. I doubt the independent blob model is applicable here.

\section{3) Icke (comment only)}

C. Regarding the wide angle tails: I believe one should say that their existence proves that the $\mathrm{CD}$ galaxies are moving with respect to something. And, if only $10 \%$ of cluster mass is in galaxies, it's not at all implausible that even a $c D$ galaxy can move merrily with respect to the overall centre of mass.

\section{4) Icke (comment)}

C. Recently, the Nagoya group has come out with a model for 3C75, along the lines that Blandford and I established for $3 C 31$ and $3 C 129$, and obtained a fairly satisfactory fit.

\section{5)Meyer}


Q. Could you comment on multi-core structure of these radio sources? (Perhaps the existence of several different active cores within one galaxy could alleviate statistical problems with the number of superluminal sources).

A. $3 C 75$ is the only source I know of in which both nuclei of a double-nucleus galaxy are radio-loud (and jet producers, to boot). Furthermore, the data certainly rule out superluminal sources being products of multiple active nuclei.

\section{6) Narasimha}

Q. Do the superluminal knots in $3 \mathrm{C} 120$ show brightness variability?

A. Their brightness certainly decreases as they move outwards from the core. I am not aware of any evidence that they re-brighten at any time. However, it is important to note that good measures of such effects are not possible yet, and must await the VLBA, or other VLBI networks which will include much better coverage in the spatial frequency plane.

\section{7) Norman}

Q. Could you say a few words about what we stand to learn as we explore the new radio bands at millimeter and meter wavelengths?

A. This is a question whose answer could fill a book. I will confine my brief remarks to extragalactic jets. It is unlikely that we will ever be able to detect the large-scale jets at millimeter wavelengths - the spectrum of the emission is such that there will be insufficient brightness. The exciting prospect at these bands is the increased resolution they bring to VLBI, perhaps eventually opening the door to resolving the activity in the nuclear core. At present, this crucial region is about 2 orders of magnitude under our best resolution on favorable (close) sources. At meter wavelengths, perhaps the best prospects are in better understanding the extensive lobes and halos, which represent the collection of end products of the jets, and in the depolarization of the sources, in which the key to the jet density, and mass flux, lie. Considerable effort, both in technology and in computing, will be required to make these bands useable, but I believe the solutions are not far off.

\section{8)Owocki}

Q. Would you comment on the observational similarities and/or differences between these AGN jets and stellar jets seen in our own galaxy? A. The most obvious difference is in scale. The AGN jets are immensely more energetic, and propagate to enormously larger scales. The internal energetics must also be different, for AGN jets are seen by nonthermal emission, while stellar jets emit through thermal processes. No spectral features from within an AGN jet are yet known, while for stellar jets, virtually all our observations are of atomic and molecular lines. The majority of the bipolar flows show much less collimation than AGN jets, although the optically detected flows discussed by Reinhart are very well collimated. 